\title{
Optimal management of giant cell arteritis and polymyalgia rheumatica
}

This article was published in the following Dove Press journal:

Therapeutics and Clinical Risk Management

4 April 2012

Number of times this article has been viewed

\section{Rodger Charlton}

College of Medicine, Swansea University, Wales, UK
Correspondence: Rodger Charlton College of Medicine, Grove Building, Swansea University, Swansea SA2 8PP, Wales, UK

Email r.c.charlton@swansea.ac.uk
Abstract: Giant cell arteritis (GCA) and polymyalgia rheumatica (PMR) are clinical diagnoses without "gold standard" serological or histological tests, excluding temporal artery biopsy for GCA. Further, other conditions may mimic GCA and PMR. Treatment with 10-20 mg of prednisolone daily is suggested for PMR or 40-60 mg daily for GCA when temporal arteritis is suspected. This ocular involvement of GCA should be treated as a medical emergency to prevent possible blindness and steroids should be commenced immediately. There are no absolute guidelines as to the dose or duration of administration; the therapeutics of treating this condition and the rate of reduction of prednisolone should be adjusted depending on the individual's response and with consideration of the multiple risks of high-dose and long-term glucocorticoids. Optimal management may need to consider the role of low-dose aspirin in reducing complications. Clinicians should also be aware of studies that indicate an increased incidence of large-artery complications with GCA. This clinical area requires further research through future development of radiological imaging to aid the diagnosis and produce a clearer consensus relating to diagnosis and treatment.

Keywords: arteritis, visual loss, blindness, erythrocyte sedimentation rate, stiffness, pain, aspirin, disability, glucocorticoids

\section{Introduction}

Giant cell arteritis (GCA) is linked with polymyalgia rheumatica (PMR). The overlap is considerable as $16 \%-21 \%$ of patients with PMR have GCA on temporal artery biopsy and symptoms of PMR are present in $40 \%-60 \%$ of patients with GCA. ${ }^{1}$ The peak incidence is between 60 and 75 years of age and incidence is increasing as the population ages. There is continuing controversy in specialist rheumatology and primary care as the optimal diagnosis and management of GCA are difficult, not least because of the syndrome's presentation as temporal arteritis (TA) with its ocular involvement, and the risk of blindness if it is untreated or under-treated. GCA is a syndrome of systemic inflammation that accompanies vascular manifestations such as TA, also referred to as cranial arteritis. Arterial biopsies may reveal changes to the tunica media vasorum and tunica adventitia that cause narrowing or occlusion of the vessel, leading to ischemia distal to the lesion.

Although PMR is a common disorder, systematic studies of its pathophysiology, diagnosis, and treatment are limited. ${ }^{2}$ It has been argued that PMR and TA are clinical syndromes that form part of the spectrum of GCA, and so are different manifestations of the same disease process. ${ }^{3,4}$ Further, it has been suggested that a generic term of "polymyalgia arteritica" should be used where the "relatively benign" PMR may later 
progress to GCA with its "multi-system pathological process" including TA. ${ }^{5}$ Although GCA, including TA, is linked with PMR, both conditions can occur in isolation from each other. ${ }^{6}$ The frequency with which each of these conditions presents differ in each source consulted, emphasizing the difficulties and lack of consensus relating to diagnosis.

PMR is an inflammatory disease that usually presents in primary care with symmetrical aching, tenderness, and stiffness of the proximal muscles of the neck, shoulders, and pelvis, particularly in the morning, which may prevent a patient getting out of bed. Muscle strength is not usually impaired but is hindered by pain. ${ }^{7}$ Frequently, it is a diagnosis of exclusion based on a syndrome of symptoms and signs where greater clarity of diagnosis is helped through some basic investigations. Distinguishing GCA and PMR is important because GCA can lead to blindness through ocular ischemia/ anterior ischemic optic neuropathy and requires higher doses of glucocorticoid medication. Approximately $10 \%$ of patients initially presenting with PMR will have vasculitis on biopsyfor example, of a temporal artery - requiring a revision of their diagnosis. The standard treatment for PMR with low-dose steroids unfortunately has no prophylaxis against the blinding that can result from ocular involvement of GCA. ${ }^{8}$

This study now considers the optimal management (diagnosis and treatment) of GCA and so builds on previous publications by the author.9,10

\section{Definitions}

Although the histological definition of "GCA" is clearer, it overlaps that of "PMR," reinforcing a statement in a study as long ago as $1938^{11}$ on the topic of TA, which refers to the condition of "rheumatic arteritis" and notes that "there is great difficulty in making a confident separation of some of the forms of arteritis into groups on account of the lack of knowledge about the causation."

The term "PMR" has an interesting history. It was not until 1957 that Barber tentatively suggested the term "polymyalgia rheumatic" for this collection of symptoms and signs. ${ }^{12}$ Prior to this, descriptions of the same clinical picture were given names such as "anarthritic rheumatoid disease" or "myalgic syndrome of the aged with systemic reaction."13

\section{Attempting to distinguish PMR from GCA}

GCA can be difficult to distinguish from, or may coexist with, PMR, and can have the complications of blindness from its effect on the ophthalmic arteries (or short posterior ciliary arteries, which are branches of the ophthalmic artery) and cardiac ischemia through its effects on the coronary arteries. ${ }^{14}$ One study makes this point well by suggesting that GCA could be an "iceberg disease" with obvious classical forms distracting attention from the "submerged mass of illness." 15 This study emphasizes the importance of palpation of the temporal arteries and auscultation of main arteries for bruits in the routine assessment of such patients. Cheung and Richards ${ }^{16}$ cite Strachan et $\mathrm{al}^{14}$ and their proposed clinical classification of GCA to improve the awareness of the diversity of this condition. It is suggested that there is "classic GCA" and what is described as "masked GCA." In masked GCA, patients can present with:

- Weight loss and cachexia

- Pyrexia of unknown origin

- Anemia

- Aortic regurgitation

- Ruptured aortic aneurysm

- Cerebrovascular accident, myocardial ischemia, or intermittent claudication (“occlusive group").

\section{Etiology}

In the UK, GCA occurs in 2.2 per 10,000 patient years. It is very unlikely to occur in people aged $<50$ years, and there is a peak incidence between the ages of 60 and 75 years. ${ }^{17}$ The disease is almost always confined to Caucasians, with a higher incidence in Scandinavia and northern Europe. The incidence varies between 10 and 50 cases/100,000 people aged $>50$ years. ${ }^{3}$ The number of people at risk is expected to double in the next 25 years as the average age of the population increases. ${ }^{18}$ The cause of PMR is unknown, as is the relationship between PMR and GCA. In a seminar on the subject, it was reported that "few population-based studies have assessed the epidemiological aspects of polymyalgia rheumatica because there is a lack of a diagnostic hallmark and universally accepted diagnostic and classification criteria."19

As the onset of symptoms can be quite sudden, a possible viral etiology has been proposed. However, "a well-defined infectious agent has never been found." 19 Genetic factors appear to be important and HLA-DR4 has been associated with PMR, but the etiology remains unclear. ${ }^{19}$

\section{Symptoms}

Criteria for GCA issued by the American College of Rheumatology in 1990 include three or more of the following ${ }^{20}$ :

- Age 50 years or older

- New-onset localized temporal headache

- Temporal artery tenderness or decreased temporal artery pulse 
- Erythrocyte sedimentation rate (ESR) of at least $50 \mathrm{~mm} / \mathrm{h}$

- Abnormal artery biopsy specimen characterized by mononuclear infiltration or granulomatous inflammation There may also be: ${ }^{21}$

- Myalgia

- Systemic malaise

- Fever

The presentation varies and may include fever of unknown origin, visual loss, or limb claudication. The arterial lesions may be widespread, hence the different manifestations and presentations that vary according to the anatomy of the area affected. Indeed, the lesions may be more widespread than the ophthalmic artery and branches of the external carotid system. GCA and PMR can share similar systemic symptoms including tiredness, fever, and weight loss, and likely represent two parts of a disease spectrum. However, GCA is at the more severe end of this spectrum.

GCA most commonly affects the branches of the internal and external carotid arteries, which can lead to symptoms such as headache or symptoms of the associated anatomy such as pain while chewing, jaw, sinus, or tongue pain. ${ }^{18}$ In GCA, inflammation in the walls of the smaller vessels leads to a narrowing of the lumen and eventual occlusion where pain is a result of ischemia. Jaw claudication and headache occur in $30 \%-80 \%$ of cases, and visual disturbances such as amaurosis fugax, hallucinations, diplopia, or irreversible visual loss occur in $<20 \%$ of cases. ${ }^{22}$

For dentists, GCA should be considered in the differential diagnosis if a patient has puzzling symptoms that are not explained by oral findings. ${ }^{23}$ GCA often manifests as a newonset headache or a headache different from previous headaches. If the patient has a headache, particularly a temporal headache, or of they develop visual symptoms or scalp tenderness, GCA affecting the extracranial arteries (eg, the temporal arteries) should be considered. A temporal artery biopsy may be indicated to confirm the diagnosis through the presence of giant cells. However, glucocorticoids should be commenced urgently with this degree of suspicion to prevent potential loss of vision.

The loss of vision in TA results from ischemia and infarction of the optic nerve, which causes the appearance of a pale and swollen optic nerve head on fundoscopy. ${ }^{8}$ Appreciable visual loss occurs in $30 \%-50 \%$ of patients with untreated GCA, but it is very difficult to predict which patients with GCA will go on to develop ocular complications. ${ }^{24}$ Occasionally, large arteries are also affected when conditions such as aortic dissection have been reported. ${ }^{25}$ Clinicians should consider the potential complications of large-artery disease to ensure optimal management.
Like GCA, the onset of PMR is usually acute. However, symptoms are generally present for longer than a month before patients seek advice. Many authors refer to "proximal girdle involvement," and the word "girdle" may be interpreted as muscles that encircle the neck, shoulders, or pelvis. Two cardinal features of PMR are girdle pain and morning stiffness lasting for over 30 minutes. $^{7}$ The aching pain and stiffness is usually of sudden onset and quickly becomes bilateral. There may also be systemic symptoms such as low-grade fever, fatigue, and weight loss. Transient peripheral synovitis of the wrists, knees, and sternoclavicular joints has also been documented. ${ }^{6}$ The presentation of PMR may therefore be symmetrical aching, tenderness, and stiffness of the proximal muscles of the neck, shoulders, and pelvis. These symptoms may be particularly pronounced in the morning and may prevent a patient from getting out of bed. Muscle strength is usually not impaired, but is hindered by pain.

\section{Difficulty of TA diagnosis}

A diagnosis of TA can easily be missed. A 1971 Swedish study of 1097 consecutive autopsies included a temporal artery examination in each autopsy and 16 cases of undiagnosed GCA were identified. ${ }^{21}$ A retrospective analysis of the medical records provided evidence of typical features of undiagnosed GCA in nine. ${ }^{26}$ In addition, an audit of 65 patients with GCA found that 44 had had unrecognized visual disturbance, visual loss, or stroke a mean of 35 days between the onset of symptoms and diagnosis (range of 2 to 336 days). ${ }^{27}$ Eleven of these patients presented without headache or scalp tenderness and ten of these had visual loss.

A meta-analysis from the $\mathrm{USA}^{28}$ was reported in a study that looked at studies examining the value of individual clinical features in predicting positive results of temporal artery biopsy in patients with suspected GCA. ${ }^{21}$ Several symptoms were moderately predictive of a positive biopsy result (likelihood ratio $>2$ ):

- Jaw claudication (34\%) (it is recorded that claudicant pain comes on gradually during chewing, whereas dental pain is immediate)

- Diplopia (8\%)

- Any abnormality on palpation of the temporal artery absent, beaded, tender, or enlarged (65\%) Other useful predictive features (likelihood ratio $>1.5$ ) were:

- Temporal headache

- Scalp tenderness

- $\quad \mathrm{ESR}>100 \mathrm{~mm} / \mathrm{h}$

- Anemia 


\section{Differential diagnosis}

Many conditions may mimic the symptoms of PMR. For example, fibromyalgia, osteoarthritis, rheumatoid arthritis, or systemic lupus erythematosus should be considered. Similarly, the possibility of an occult malignancy or sepsis that can present with muscle pains should form part of the differential diagnoses. Further, a combination of osteoarthritis with a systematic problem such as intercurrent infection may appear to be PMR. ${ }^{6}$ Metabolic conditions should be excluded, including thyroid disease and hyperparathyroidism. The likelihood of such conditions is greater with a normal ESR on presentation, a poor response to corticosteroids, age of onset $<50$ years, an absence of upper limb involvement, and a slow onset. PMR is currently a clinical diagnosis without a "gold standard" serological or histological test. ${ }^{6}$

This study will also consider the development of radiological imaging that may benefit the diagnostic process in the future.

\section{Parameters for diagnosis}

A raised ESR continues to be the universal diagnostic parameter and the parameter by which response to treatment with steroids is judged and the condition monitored. The "lack of a gold standard bedevils the establishment" of an accurate diagnosis. ${ }^{29}$ If it appears that, clinically, a patient has PMR, C-reactive protein (CRP) should be measured as this may be raised in patients with a normal ESR. Measurement of the plasma viscosity (PV) may also be considered. ${ }^{30,31}$ The results of studies vary, but an ESR of at least $40 \mathrm{~mm} / \mathrm{h}$ supports the diagnosis with associated symptoms. However, $5 \%-20 \%$ of cases may have an ESR within the normal range. ${ }^{16,19}$

There may be a mild normocytic anemia, which is frequently associated with chronic disease, but tests for rheumatoid factor and antinuclear antibodies are generally negative. A rapid response to steroids is an important diagnostic pointer. ${ }^{3,7}$ In GCA, arteries may be thickened, tender, and nodular, with pulsation being absent or reduced. ${ }^{17}$ Temporal artery biopsy is not an investigation with a high sensitivity and where GCA is suspected, despite a negative biopsy, steroid treatment should be started. ${ }^{5,17}$ Also, it is difficult to obtain biopsies and biopsy results quickly. ${ }^{17}$

\section{Mainstay of treatment}

Glucocorticoids are the drug of choice. If GCA is also suspected, Salvarani and colleagues suggest a dose of 40-60 mg of prednisolone daily, as this is the dose necessary to suppress the disease, and a dose of 10-20 mg daily of prednisolone for PMR. ${ }^{18,19}$ In GCA, if the patient has ischemic symptoms (jaw or tongue claudication, or visual symptoms), $60 \mathrm{mg}$ prednisolone daily should be given. If the patient has visual symptoms, they should be admitted to hospital for treatment with intravenous methylprednisolone. ${ }^{21}$ Although there are no widely accepted standard treatment schedules in relation to the dose or its duration, there are helpful British Society for Rheumatology (BSR) and British Health Professionals in Rheumatology (BHPR) guidelines. ${ }^{32}$ Essentially, the amount of prednisolone can be reduced once symptoms have eased (which they usually do quickly) and the ESR has normalized. If symptoms start to relapse, then the reduction in dose has been too soon.

\section{Clinical risk management}

A patient presenting with sudden loss of vision in one or both eyes should be given an intravenous injection of $10 \mathrm{mg}$ dexamethasone because of the risk of permanent blindness. ${ }^{5}$ Treatment with glucocorticoids is mandatory for GCA to prevent vascular complications and treatment may be required for 3-4 years, with relapses most likely in the first 18 months of treatment. Relapses may occur in a third or more of patients and should be treated by a dose of prednisolone the same or greater than that given before relapse, depending on the severity of symptoms. ${ }^{17}$ Relapse is associated with rapid tapering of the dose; ${ }^{6}$ to prevent this, where a patient is on $15 \mathrm{mg}$ (for example), this should be maintained for 4 weeks and then reduced to $12.5 \mathrm{mg}$ for another 4 weeks. When a dose of $10 \mathrm{mg}$ is reached, it should be reduced by $1 \mathrm{mg}$ every 4-8 weeks, with the rate of reduction adjusted depending on the response. The dose, length of treatment, and rate of reduction are based largely on observational studies. There should, however, be a dramatic response to treatment within a few days, and tapering of the dose should be individualized. ${ }^{6,18}$ The BSR and BHPR guidelines should be consulted for optimal treatment. ${ }^{32}$

There are many potential side effects of glucocorticoids, which, paradoxically, can lead to weakness rising from sitting, or falls. ${ }^{18}$ The following list of side effects demonstrates how it is important to monitor blood glucose with ESR measurements and to put in place measures to prevent osteoporosis. Side effects of treatment with glucocorticoids may include: Acute

- Risk of peptic ulceration is increased in patients who are concurrently receiving nonsteroidal anti-inflammatory drugs or aspirin

- Infection through suppression of the immune response

- Reduced potassium leading to muscle weakness

- Increased sodium 
- Sleep disturbance

- Confusional states

Long-term

- Osteoporosis

- Addisonian crisis with rapid withdrawal

- Reduced glucose tolerance and diabetes

- Corticosteroid myopathy

Patients should be made aware of possible side effects of glucocorticoids and should alert other health care practitioners that they are on glucocorticoids, as there will be an increased risk of infection or gastrointestinal bleeding. As patients may be on glucocorticoids for some time, it is appropriate to consider prophylaxis with calcium and vitamin $\mathrm{D}$, as well as other treatment, such as a bisphosphonate for those found to have osteopenia or osteoporosis. Ideally, concomitant bisphosphonate and calcium/vitamin D therapy should be prescribed when glucocorticoid treatment begins. This is particularly important because GCA and PMR may occur in older people who are already at greater risk of osteoporosis. Helpful guidelines for the management of glucocorticoidinduced osteoporosis are provided in the 2010 American College of Rheumatology recommendations. ${ }^{33}$

An alternative treatment is intramuscular or intravenous methylprednisolone acetate, for which a lower incidence of glucocorticoid-related side effects has been documented. ${ }^{6}$ If intramuscular or intravenous methylprednisolone pulse therapy is administered, there is evidence of reduced incidence of GCA complications, including visual loss. ${ }^{34}$ In addition, the use of pulse therapy in the initial treatment of GCA allows for a more rapid tapering of oral glucocorticoids, less likelihood of relapse, and reduced potential for the long-term side effects of treatment with oral glucocorticoids. ${ }^{35}$

The use of glucocorticoid-sparing agents, such as methotrexate, azathioprine, cyclophosphamide, and cyclosporine, may be useful in reducing the side effects of glucocorticoids. However, due to a lack of data, no conclusive recommendations can be made. ${ }^{23}$

Cerebrovascular accidents and visual loss are potential causes of morbidity with GCA and are due to cranial ischemia. One study suggests that the use of low-dose aspirin in addition to corticosteroid therapy may lessen the risk of these complications, and this is recommended by the European League Against Rheumatism. ${ }^{36}$

\section{Duration of treatment}

As relapses are more likely to occur during the initial 18 months of therapy and within 1 year of glucocorticoid withdrawal, all patients should be monitored for symptom recurrence throughout corticosteroid tapering and until 12 months after therapy cessation. Approximately 50\%-75\% of patients can discontinue glucocorticoid therapy after 2 years of treatment. This is a controversial area of rheumatological practice, as it is an ongoing challenge - no consensus has been reached about optimal management and further research is required.

\section{Outcomes of disease process and prognosis}

GCA and PMR are self-limiting conditions that usually last 2 years, but there have been reports of cases persisting for up to 7 years. ${ }^{37}$ It was reported in 1979 that "the difficulty often lies not in starting treatment but in deciding how long it should continue." ${ }^{38}$ There is a need for a careful assessment to maintain an acceptable balance between the benefits and risks of long-term glucocorticoid therapy. ${ }^{25}$ Acute blindness occurs in up to $20 \%$ of patients with GCA. ${ }^{4}$ Delay in recognition may explain the high incidence of irreversible loss of vision, which is preventable with early diagnosis and treatment. ${ }^{21}$

Optimal management of PMR and GCA should take into account studies indicating an increased incidence of large-artery complications including aortic aneurysm, aortic dissection, and large-artery stenosis. In the USA, one population-based study from 1950 over 50 years identified 46 cases of large-artery complication in 168 patients, which represents $27 \%$ of the cohort studied. ${ }^{39}$ It has been suggested that large-artery involvement is common in GCA and that early intervention with glucocorticoid therapy is appropriate. $^{40}$

\section{Future developments}

Future diagnostic techniques may be enhanced by technological improvements in radiological imaging. In PMR, routine $\mathrm{X}$-rays do not usually reveal any abnormalities. However, magnetic resonance imaging (MRI) has demonstrated that PMR involves inflammation of extra-articular synovial structures and, in particular, that MRI scans of the shoulder show bilateral subacromial and subdeltoid bursitis in nearly all patients with active PMR. ${ }^{41}$ Similarly, ultrasonography often reveals effusions within both shoulder bursae in patients with PMR. ${ }^{42}$

The role of positron emission tomography (PET) scanning is less clear but may indicate that subclinical inflammation of the great vessels is more common than previously supposed in PMR. ${ }^{43}$ The sensitivity and specificity and, thus, interpretations of the findings from these investigations 
in relation to GCA need to be agreed and validated. It has been suggested that these investigations will lead to "a new era where our understanding of the extent of vascular involvement in GCA may become more precise." 44 This has implications for both diagnosis and management, subject to the ready availability of these investigations, their speed, and their affordability.

\section{Conclusion}

Few population-based studies have assessed the epidemiological aspects of GCA and PMR because there is a lack of a diagnostic hallmark and universally accepted diagnostic and classification criteria. There remain many unknowns in the diagnosis and treatment of GCA, and its overlap with PMR. It remains a challenge and an area requiring further research. However, appropriate use of glucocorticoids remains the cornerstone of treatment. $22,32,33$

\section{Acknowledgments}

The author wishes to acknowledge Dr RW Strachan, Emeritus Consultant Physician, Dumfries and Galloway Royal Infirmary, Scotland, who was a stimulus to his interest in this area when he was a junior house officer in 1984. The author also wishes to acknowledge his previous work in this area in preparation for a publication ${ }^{9}$ in Clinical Medicine in 2008 .

\section{Disclosure}

The author reports no conflicts of interest in this work.

\section{References}

1. Salvarani C, Cantini F, Hunder GG. Polymyalgia rheumatica and giantcell arteritis. Lancet. 2008;372(9634):234-245.

2. Matteson EL. Clinical guidelines: unravelling the tautology of polymyalgia rheumatica. Nat Rev Rheumatol. 2010;6(5):249-250.

3. Swannell AJ. Polymyalgia rheumatica and temporal arteritis: diagnosis and management. BMJ. 1997;314(7090):1329.

4. Smeeth L, Cook C, Hall AJ. Incidence of diagnosed polymyalgia rheumatica and temporal arteritis in the United Kingdom, 1990-2001. Ann Rheum Dis. 2006;65(8):1093-1098.

5. Sudlow C. Diagnosing and managing polymyalgia rheumatica and temporal arteritis. Sensitivity of temporal artery biopsy varies with biopsy length and sectioning strategy. BMJ. 1997;315(7107):549.

6. Sengupta R, Kyle V. Recognising polymyalgia rheumatica. Practitioner. 2006;250(1688):40-44, 47.

7. Currey J. Polymyalgia rheumatica and giant cell arteritis. Update. 1989;52:37-41.

8. Durkin SR, Athanasiov PA, Crompton JL. Polymyalgia rheumatica and giant cell arteritis - an ophthalmic emergency. Aust Fam Physician. 2006;35(11):889-891.

9. Charlton R. Polymyalgia rheumatica and its links with giant cell arteritis. Clin Med. 2008;8(5):498-501.
10. Charlton R. A continuing controversy in rheumatology - optimal diagnosis and management of polymyalgia rheumatica and its links with giant cell arteritis. Poster presentation at CORA International Conference, Italy, March 10-12, 2011. Abstract published in Clinical and Experimental Rheumatology. 2011;29(1):206.

11. Jennings GH. Arteritis of the temporal vessels. Lancet. 1938; 231(5973):424-428.

12. Barber HS. Myalgic syndrome with constitutional effects; polymyalgia rheumatica. Ann Rheum Dis. 1957;16(2):230-237.

13. Polymyalgia rheumatica. Lancet. 1961;277(7177):597-599.

14. Strachan RW, How J, Bewsher PD. Masked giant-cell arteritis. Lancet. 1980;315(8161):194-196

15. How J, Strachan RW, Bewsher PD. Giant cell arteritis - a cardiological blind spot? Am Heart J. 1980;100(3):405-407.

16. Cheung PP, Richards G. Masked giant cell arteritis. Aust Fam Physician. 2006;35(11):893-895.

17. Pountain G, Hazleman B. ABC of rheumatology. Polymyalgia rheumatica and giant cell arteritis. BMJ. 1995;310(6986):1057-1059.

18. Unwin B, Williams CM, Gilliland W. Polymyalgia rheumatica and giant cell arteritis. Am Fam Physician. 2006;74(9):1547-1554.

19. Salvarani C, Macchioni P, Boiardi L. Polymyalgia rheumatica. Lancet. 1997;350(9070):43-47.

20. Hunder GG, Bloch DA, Michel BA, et al. The American College of Rheumatology 1990 criteria for the classification of giant cell arteritis. Arthritis Rheum. 1990;33(8):1122-1128.

21. Hassan N, Dasgupta B, Barraclough K. Giant cell arteritis. BMJ. 2011;342:d3019.

22. Luqmani R. Treatment of polymyalgia rheumatica and giant cell arteritis: are we any further forward? Ann Intern Med. 2007;146(9):674-676.

23. Allen JC. Temporal arteritis: don't let this disease fool you. Gen Dent. 2007;55(1):52-53.

24. Ferris J, Lamb R. Polymyalgia rheumatica and giant cell arteritis. High dose corticosteroids are recommended. BMJ. 1995;311(7002):455.

25. Kalke $\mathrm{S}$, Dasgupta B. Polymyalgia rheumatica and giant cell arteritis. Update. 2002;65(1):22-27.

26. Ostberg G. Temporal arteritis in a large necropsy series. Ann Rheum Dis. 1971;30(3):224-235.

27. Ezeonyeji AN, Borg FA, Dasgupta B. Delays in recognition and management of giant cell arteritis: results from a retrospective audit. Clin Rheumatol. 2011;30(2):259-262.

28. Smetana GW, Shmerling RH. Does this patient have temporal arteritis? JAMA. 2002;287(1):92-101.

29. Ortiz Z, Tugwell P. Raised ESR in polymyalgia rheumatica no longer a sine qua non? Lancet. 1996;348(9019):4-5.

30. Murphy PT, Wood JK. Polymyalgia rheumatica. Lancet. 1996; 348:550.

31. Hopayian K. Diagnosing polymyalgia rheumatica. Lancet. 1996; 348(9031):899.

32. Dasgupta B, Borg F, Hassan N, et al. BSR and BHPR guidelines for the management of polymyalgia rheumatica. Rheumatology. 2010;49(1):186-190.

33. Grossman JM, Gordon R, Ranganath VK, et al. American College of Rheumatology 2010 recommendations for the prevention and treatment of glucocorticoid-induced osteoporosis. Arthritis Care Res (Hoboken). 2010;62(11):1515-1526.

34. Weinberg DA, Savino PJ, Sergott RC, Bosley TM. Giant cell arteritis, Corticosteroids, temporal artery biopsy, and blindness. Arch Fam Med. 1994;3(7):623-627.

35. Mazlumzadeh M, Hunder GG, Easley KA, et al. Treatment of giant cell arteritis using induction therapy with high-dose glucocorticoids: a double-blind, placebo-controlled, randomized prospective clinical trial. Arthritis Rheum. 2006;54(10):3310-3318.

36. Nesher G, Berkun Y, Mates M, Baras M, Rubinow A, Sonnenblick M. Low-dose aspirin and prevention of cranial ischemic complications in giant cell arteritis. Arthritis Rheum. 2004;50(4):1332-1337. 
37. Kyle V, Hazelman BL. Stopping steroids in polymyalgia rheumatica and giant cell arteritis. BMJ. 1990;300(6721):344-345.

38. Steroids in polymyalgia rheumatica. Lancet. 1979;314(8138):341.

39. Nuenninghoff DM, Hunder GG, Christianson TJ, McClelland RL, Matteson EL. Incidence and predictors of large-artery complication (aortic aneurysm, aortic dissection, and/or large-artery stenosis) in patients with giant cell arteritis: a population-based study over 50 years. Arthritis Rheum. 2003;48(12):3522-3531.

40. Matteson EL. Large vessel involvement in giant cell arteritis: incidence, predictors and mortality. Conference Abstract OP 24. Third International Conference on Giant Cell Arteritis and Polymyalgia Rheumatica. Rheumatology. 2005;44(Suppl 3):iii10-iii11.

41. Hunder GG. Clinical manifestations and diagnosis of polymyalgia rheumatic [article on the Internet]. UpToDate. Topic 8235, version 5.0. Waltham, MA: UpToDate Inc; 2010 [last updated Feb 3]. Available from: http://www.uptodate.com/contents/clinical-manifestations-anddiagnosis-of-polymyalgia-rheumatica. Accessed March 22, 2012.
42. Cantini F, Salvarani C, Olivieri I, et al. Shoulder ultrasonography in the diagnosis of polymyalgia rheumatica: a case-control study. J Rheumatol. 2001;28(5):1049-1055.

43. Moosig F, Czech N, Mehl C, et al. Correlation between 18-fluorodeoxyglucose accumulation in large vessels and serological markers of inflammation in polymyalgia rheumatica: a quantitative PET study. Ann Rheum Dis. 2004;63:870-873.

44. Cid MC, Prieto-González S, Arguis P, et al. The spectrum of vascular involvement in giant-cell arteritis: clinical consequences of detrimental vascular remodelling at different sites. APMIS Suppl. 2009;117(s127):10-20.

\section{Publish your work in this journal}

Therapeutics and Clinical Risk Management is an international, peerreviewed journal of clinical therapeutics and risk management, focusing on concise rapid reporting of clinical studies in all therapeutic areas, outcomes, safety, and programs for the effective, safe, and sustained use of medicines. This journal is indexed on PubMed Central, CAS,

\section{Dovepress}

EMBase, Scopus and the Elsevier Bibliographic databases. The manuscript management system is completely online and includes a very quick and fair peer-review system, which is all easy to use. Visit http://www.dovepress.com/testimonials.php to read real quotes from published authors.

Submit your manuscript here: http://www.dovepress.com/therapeutics-and-clinical-risk-management-journal 